\title{
Early vitrectomy for progressive diabetic proliferations covering the macula
}

\author{
Ralf Grewing, Ulrich Mester
}

\begin{abstract}
The clinical course in $\mathbf{5 0}$ eyes was analysed after pars plana vitrectomy for progressive diabetic fibrovascular proliferations. Patients were assigned to pars plana vitrectomy if progression of proliferations occurred despite a photocoagulation treatment with a mean number of 3500 burns and additional peripheral cryoablation. All cases had visual impairment because of fibrovascular tissue covering the macula without detachment of the macula. Flat proliferations were present in all eyes without retinal elevation, vitreous detachment, or vitreous haemorrhage. The follow up intervals ranged from 13 months to 39 months (mean interval 24 months). Twelve months postoperatively, 36 eyes $(72 \%)$ showed improved visual acuity, five eyes $(10 \%)$ were worse, and nine eyes $(18 \%)$ were unchanged. Thirty two eyes (64\%) achieved a final visual acuity of 0.2 or better, and 45 eyes $(90 \%)$ gained $\mathbf{0 . 0 5}$ or better. In only two eyes could reproliferation be observed. The postoperative course indicates that pars plana vitrectomy for diabetic fibrovascular proliferations covering the macula can preserve socially useful visual acuity of at least $\mathbf{0 . 0 5}$ in most cases.
\end{abstract}

(Brf Ophthalmol 1994; 78: 433-436)

Panretinal photocoagulation (PRP) is an effective treatment for advanced stages of diabetic retinopathy. However, the Early Treatment Diabetic Retinopathy Study (ETDRS) showed that even after panretinal photocoagulation almost $20 \%$ of eyes developed a high risk proliferative retinopathy. ${ }^{1}$

If no regression of fibrovascular proliferations occurs, it is widespread clinical practice to apply further photocoagulation and/or cryocoagulation. Despite an additional laser photocoagulation of up to 7500 burns $11 \%$ to $22 \%$ of these eyes deteriorated in visual acuity to counting fingers or less. ${ }^{23}$ Vine $^{2}$ concluded that eyes without a satisfactory response after PRP of 3000 burns are presumably not going to respond to treatment.

Consequently we started to vitrectomise such eyes with proliferative diabetic retinopathy if progression of fibrovascular tissue could be observed after a PRP of a mean number of 3500 burns and additional peripheral cryotherapy. We did not wait for macular detachment or vitreous haemorrhage to occur.

\section{Materials and methods}

Patients with proliferative diabetic retinopathy were entered into this study if the fibrovascular proliferations progressed despite PRP with an average spot number of $3500(2800-4100)$ and peripheral cryoablation. The indication for PRP was severe non-proliferative or proliferative diabetic retinopathy according to the definitions of the ETDRS. ${ }^{1}$ In fluorescein angiography before PRP, five eyes (10\%) showed non-proliferative diabetic retinopathy, but non-perfusion areas were pronounced in the mid-periphery. According to the treatment protocol of the ETDRS full scatter photocoagulation with a total of 1200 to 1600 argon laser burns was applied. ${ }^{4}$ Despite PRP neovascular proliferations developed in the five eyes which had shown only severe nonproliferative diabetic retinopathy before PRP. In the other cases progression of the neovascular networks occurred. Therefore, additional photocoagulation, with a minimum of 2800 burns of spot size $0.5 \mathrm{~mm}$ and exposure time 0.1 second as well as peripheral cryocoagulation, was performed. The laser power was adjusted to obtain moderately intense white burns that do not spread to become appreciably larger than $500 \mu \mathrm{m}$.

In all cases included into this study progression of new vessels occurred. Consequently these eyes were assigned to pars plana vitrectomy.

Apart from progression of neovascularisations and despite extensive laser photocoagulation treatment and peripheral cryotherapy, further entry criteria had to be fulfilled: eyes were eligible for entry if flat fibrovascular tissue covering the macula had led to visual impairment without significant detachment of the macula. Also absence of posterior vitreous detachment, traction retinal detachment, and vitreous haemorrhage were mandatory. Decrease in visual acuity was not due to tractional detachment of the macula but was caused by the fibrovascular tissue. This may be explained by the opacity of the proliferative membranes and/ or impairment of the blood-retina barrier in the macular area. All removed proliferations consisted of active new vessels and fibrous tissue. Progression of fibrovascular tissue was given if two observers (RG and UM) congruently observed an enlargement of the fibrovascular tissue, documented at two consecutive visits. Photodocumentation was not performed routinely.

The technique for surgical management consisted of en bloc resection of the posterior hyaloid and vasoproliferative tissue. Scissors were used as a pick to elevate the posterior hyaloid. In all cases the posterior hyaloidal membrane was still adherent to the retina and could be removed together with the neovascular network. This surgical technique accomplishes complete removal of the posterior hyaloid because no small circumscribed remnant islands remain. 
Figure 1 Scattergram showing visual acuities before and 12 months after pars plana vitrectomy for progressive diabetic proliferations.

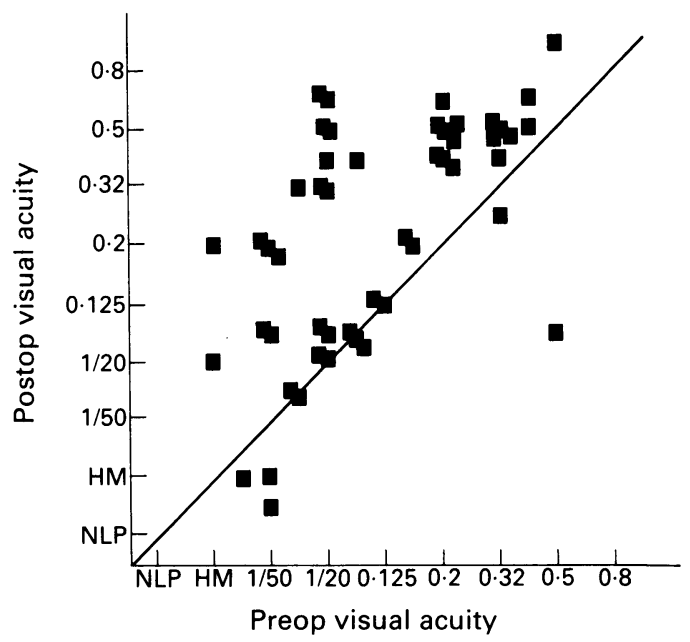

Results

The study involved 50 consecutive eyes that underwent pars plana vitrectomy for progressive fibrovascular proliferations between 3/90 and $1 / 92$. The overall rate of pars plana vitrectomies for complications of diabetic retinopathy performed during this period was 412 .

Fifty four per cent of the patients were men. The mean age was 49 years (25-71 years). The duration of diabetes was between 2 and 35 years (mean 20 years); $26 \%$ of the patients were classified according to the Diabetic Retinopathy Vitrectomy Study 5 as having type I diabetes, $46 \%$ type II diabetes, and $28 \%$ the intermediate type. Twenty four per cent showed demonstrable renal deterioration: $4 \%$ were dependent on dialysis; $20 \%$ had renal insufficiency in a compensated state. The creatinine varied between 62 and $221 \mu \mathrm{mol} / \mathrm{l}$ (mean 103).

Ten per cent of the eyes had mild neovascularisation of the iris preoperatively. Another 10\% demonstrated wide angle glaucoma before vitrectomy.

Three eyes (6\%) were pseudophakic. In $6 \%$ of the cases the lens was clear, $8 \%$ showed mild and $80 \%$ moderate lens opacities. Results are evaluated for an average follow up of 24 months (range 13-39 months). Visual acuities are given for a specific postoperative follow up of 12 months.

During pars plana vitrectomy (three port system) endodiathermy was used in $26 \%$ to stop bleeding from the neovascularisations. An iatrogenic retinal break occurred in one eye (2\%) and was successfully managed by sulphur hexafluoride gas tamponade and additional laser coagulation, applied with the indirect ophthalmoscope. In only two eyes $(4 \%)$ were mild new retinal vessels at the disc present at the final examination. No non-vascularised reproliferation could be observed during follow up. Temporary bleeding into the vitreous cavity after pars plana vitrectomy occurred in 15 eyes. A lavage procedure was performed in five eyes. The interval between vitrectomy and lavage was 3 months in two cases and 5 months in the other three cases. One patient with non-resolving haemorrhage deferred the necessary lavage. A relavage procedure had to be performed in one eye, combined with intraocular silicone oil tamponade to prevent rebleeding. In 36 eyes (72\%) final visual acuity was, at 12 months postoperatively, better than preoperatively. The visual acuity worsened in five eyes $(10 \%)$ and was unchanged in nine eyes (18\%). Thirty two eyes $(64 \%)$ achieved a final visual acuity of 0.2 or better, and 45 eyes $(90 \%)$ achieved a socially useful visual acuity ${ }^{6}$ of 0.05 or better. Detailed information of pre- and postoperative visual acuities 12 months after pars plana vitrectomy is given in Figure 1. Reasons for deterioration of visual acuity in the five eyes were atrophy of the optic nerve (one eye), acceleration of exudative maculopathy (two eyes), and macular ischaemia (one eye); one patient with persistent rebleeding into the vitreous cavity refused a lavage procedure.

Cataract formation progressed during follow up in five eyes $(10 \%)$. In one case (1/5) phacoemulsification with posterior lens implantation in the capsular bag became necessary 8 months after pars plana vitrectomy. One eye showed a mild rubeosis iridis at the final examination. No new glaucoma developed during follow up.

\section{Discussion}

All patients in our group showed progression of new vessels despite PRP preoperatively. Even additional photocoagulation up to 3500 burns and peripheral cryocoagulation did not stop the growth of fibrovascular proliferations. In these cases it is widespread clinical practice to apply further photocoagulation. ${ }^{37}$ Aylward $e t a l^{3}$ reported a final visual acuity of $6 / 18$ or better in $89 \%$ of eyes treated with a mean number of 7225 burns. In contrast to these results 11 of 23 eyes showed no satisfactory response after an average of 7550 Goldmann burns applied by Vine ${ }^{2}: 45 \%$ of these eyes had a severe decrease in visual acuity to counting fingers or less. Vine concluded that eyes that had not shown a satisfactory response after initial PRP of approximately 3000 burns were presumably not going to respond. Further inevitable side effects of confluent laser photocoagulation are the loss of visual field and the loss of colour discrimination..$^{18}$

We got the impression that eyes resistant to laser coagulation of about 3500 burns and additional peripheral cryoablation may undergo visual deterioration secondary to persistent vitreous haemorrhage, traction retinal detachment, or combined tractional/rhegmatogenous detachment as the vitreous cortex contracts. Consequently we started to vitrectomise eyes with proliferative diabetic retinopathy if the growth of fibrovascular tissue could not be stopped after a PRP of a mean number of 3500 burns and peripheral cryoablation.

As early as 1980 the Diabetic Retinopathy Vitrectomy Study (DRVS) included patients with very severe proliferative diabetic retinopathy who still retained useful vision in a randomised trial. ${ }^{10}{ }^{10}$ The advantage of vitrectomy tended to increase with increasing severity of new vessels. Two thirds of the eyes in the DRVS showed retinal elevation or preretinal haemorrhage. Other data concerning early vitrectomy in diabetic eyes have been published by Blankenship and Machemer," de Bustros et $a l,{ }^{12}$ and Shea. ${ }^{6}$ In contrast with these studies preoperative visual acuity was not an eligibility criterion 
in our patient group. Moreover, no signs of partial retinal or vitreous detachment were present. Because of these special entry criteria our results are only partially comparable with previous studies.

Intraoperative bleeding occurred in some of our cases, especially when the posterior hyaloid together with the vasoproliferative tissue were removed from the optic disc. In contrast with our experience with the membrane dissection technique, which we have used in former times, the en bloc resection technique reduces the incidence of intraoperative bleeding. Thus, endodiathermy had to be used in $26 \%$ of the operations only. This may be due to the fact that en bloc resection amputates the new vessels at the site of their origin where the new vessels have muscle cells comparable with the retinal vasculature. This may also explain the observation that bleeding from larger trunks often stops sooner than haemorrhages from smaller extensions of the neovascular network. ${ }^{13}$

Despite the fact that in our study only two eyes redeveloped new retinal vessels on the retinal surface during follow up, in 15 eyes temporary bleeding in the vitreous cavity occurred. Postoperative haemorrhages occurred predominantly in the first 6 months after vitrectomy and resolved spontaneously in most cases. A lavage procedure had to be performed in only five eyes. One of these cases needed a relavage procedure 8 months later. Focal endolaser photocoagulation became avoidable after switching from the membrane dissection technique to the en bloc resection technique. Therefore, no focal endophotocoagulation of severed epicentres was necessary.

Rebleeding into the vitreous cavity occurred in 15 eyes; in one case a relavage became necessary. Haemorrhages are a frequent complication after vitrectomy in diabetics. ${ }^{14}$ is Any bleeding during the early postoperative course tends to clear rapidly, although the rate of clearing depends on the amount of haemorrhage. A major therapeutic problem is the recurrence of vitreous haemorrhage which is a clinical feature of anterior hyaloidal fibrovascular proliferations (AHFP). ${ }^{16}$ Lewis et al used panretinal argon laser endophotocoagulation in all patients with recurrent haemorrhages due to AHFP except those who already had extensive panretinal photocoagulation before surgery. ${ }^{17}{ }^{18}$ Because all eyes included in our study had been treated with extensive panretinal photocoagulation and peripheral cryotherapy preoperatively no further intraoperative photocoagulation was performed.

Four of five eyes with preoperative rubeosis iridis showed regression after pars plana vitrectomy. Other investigations revealed an increase of iris neovascularisations after vitrectomy, especially in cases with combined lensectomy. ${ }^{19-2} 1$

Interestingly, postoperative rubeosis iridis developed in only $2 \%$ of eyes with an uncomplicated postoperative course. ${ }^{22}$ Eyes with mild or moderate rubeosis iridis were also eligible for entry in the prospective diabetic retinopathy vitrectomy study. ${ }^{10}$ But neither any correlation with the visual outcome nor progression of rubeosis iridis postoperatively are reported. The regression of mild rubeosis iridis in four of five eyes could be independent of the vitrectomy but could also be related to the extensive panretinal laser photocoagulation applied preoperatively. In a prospective study by Doft and Blankenship $36 \%$ of eyes that failed to show an initial favourable response by 3 weeks, did show a delayed improvement in low risk characteristics at 6 months. ${ }^{23}$

The anatomical alteration at the vitreoretinal interface performed by pars plana vitrectomy is comparable with a complete posterior vitreous detachment. Akiba et al reported an incidence of neovascularisations in diabetic retinopathy of only $3 \%$ in eyes with complete posterior detachment. ${ }^{24}$ This low incidence of retinal neovascularisation is comparable with the rate of proliferations after diabetic vitrectomy. Rice and Michels also report a reproliferation rate of $3 \%$ after vitrectomy for diabetic retinopathy. ${ }^{21}$ Fibrovascular proliferation seems to be reduced when the scaffold of the adjacent posterior vitreous surface is absent. ${ }^{25}$ Only two of our patients $(4 \%)$ showed neovascularisations at the posterior pole during follow up. The reason for reproliferations in-these two cases is speculative because the en bloc resection during vitrectomy has been extended to the extreme periphery using scleral indentation. This operative technique leaves no vascular epicentre islands.

In this study visual acuity improved in $72 \%$ of the eyes, worsened in $10 \%$, and was unchanged in $18 \%$. Reasons for deterioration of visual acuity were atrophy of the optic nerve (one), acceleration of exudative maculopathy (two), macular oedema (one), and rebleeding (one). All these findings can not only be attributed to the surgical procedure but could also occur without vitrectomy.

Our results suggest that pars plana vitrectomy can preserve an economically or at least socially useful visual acuity ${ }^{6}$ in $90 \%$ of the eyes which fulfil the above mentioned eligibility criteria. We do not believe that these favourable results could be achieved by an extensive confluent laser photocoagulation. But this could only be proved in a carefully monitored trial.

Presented in part at the XVIII meeting Club Jules Gonin 1992, Vienna, Austria.

1 Early Treatment Diabetic Retinopathy Study Research Group. Early photocoagulation for diabetic retinopathy. ETDRS report number 9. Ophthalmology 1991; 98: 766-85.

2 Vine AK. The efficacy of additional argon laser photocoagulation for persistent, severe proliferative diabetic retinopathy. Ophthalmology 1985; 92: 1532-7.

3 Aylward GW, Pearson RV, Jagger JD, Hamilton AM. Extensive argon laser photocoagulation in the treatment of proliferative diabetic retinopathy. Br $\mathcal{F}$ Ophthalmol 1989; 73: proliferative

4 Early Treatment Diabetic Retinopathy Study Research Group. Techniques for scatter and local photocoagulation treatment of diabetic retinopathy. ETDRS report number 3. Int Ophthalmol Clin 1987; 27: 254-64.

5 Diabetic Retinopathy Vitrectomy Study Research Group. Early vitrectomy for severe proliferative diabetic retinopathy in eyes with useful vision. Results of a randomized trial. DRVS report number 3. Ophthalmology 1988; 95: 1307-20.

6 Shea $M$. Early vitrectomy in proliferative diabetic retinopathy. Arch Ophthalmol 1983; 101: 1204-5.

7 Singerman LJ, Weaver DT. PDR in juvenile onset diabetics: high-risk proliferative diabetic retinopathy in juvenile onset diabetics. Retina 1981; 1:18-26.

8 Frank RN. Visual fields and electroretinography following extensive photocoagulation. Arch Ophthalmol 1975; 93: 591-8.

9 Kupfer C. A new patient group in the diabetic retinopathy vitrectomy study. Arch Ophthalmol 1981; 99:65.

10 Diabetic Retinopathy Vitrectomy Study Research Group. 
Two-year course of visual acuity in severe proliferative diabetic retinopathy with conventional management. DRVS report number 1. Ophthalmology 1985; 92: 492-502.

11 Blankenship GW, Machemer R. Prophylactic vitrectomy in proliferative diabetic retinopathy. Mod Probl Ophthalmo 1977; 18: $236-41$.

12 De Bustros S, Thompson JT, Michels RG, Rice TA Vitrectomy for progressive proliferative diabetic retinopathy. Arch Ophthalmol 1987; 105: 196-9.

13 Aaberg TM, Abrams GW. Changing indications and techniques for vitrectomy in management of complications of
diabetic retinopathy. Ophthalmology 1987; 94: 775-9.

14 Novak MA, Rice TA, Michels RG, Auer C. Vitreous hemorrhage after vitrectomy for diabetic retinopathy. Ophthalrhage after vitrectomy for
mology $1984 ; 91: 1485-9$.

15 Blankenship GW. Management of vitreous cavity hemorrhage following pars plana vitrectomy for diabetic retinopathy. Ophthalmology 1986; 93: 39-44.

16 Mester U, Grewing R. Recurrent postoperative hemorrhage in diabetic eyes by anterior hyaloidal proliferation. In: Stirpe $\mathrm{M}$, ed. Advances in vitreoretinal surgery. 1 st ed. New York: Ophthalmic Communications Society, 1992: 91-3.

17 Lewis H, Abrams GW, Williams GA. Anterior hyaloida fibrovascular proliferation after diabetic vitrectomy. $A m \mathcal{F}$ Ophthalmol 1987; 104: 607-13.
18 Lewis H, Abrams GW, Foos RY. Clinicopathologic findings in anterior hyaloidal fibrovascular proliferation after diabetic vitrectomy. Am $\mathcal{F}$ Ophthalmol 1987; 104: 614-8.

19 Michels RG, Rice TA, Rice EF. Vitrectomy for diabetic vitreous hemorrhage. Am $\mathcal{F}$ Ophthalmol 1983; 95: 12-21.

20 Rice TA, Michels RG, Rice AF. Vitrectomy for diabetic traction retinal detachment involving the macula. $A m \mathcal{F}$ Ophthalmol 1983; 95: 22-3.

21 Rice TA, Michels RG. Long-term anatomic and functional results of vitrectomy for diabetic retinopathy. Am $\mathcal{F}$ Ophthalmol 1980; 90: 297-303.

22 Schachat AP, Oyakawa RT, Michels RG, Rice AP. Complications of vitreous surgery for diabetic retinopathy. II. tons of vitreous surgery for diabetic retinopathy. II.
Postoperative complications. Ophthalmology 1983; 90: 522Postope

23 Doft BH, Blankenship G. Retinopathy risk factor regression after laser panretinal photocoagulation for proliferative diabetic retinopathy. Ophthalmology 1984; 91: 1453-7.

24 Akiba J, Arzabe CW, Trempe CL. Posterior vitrous detachment and neovascularization in diabetic retinopathy. Ophthalmology 1990; 97: 889-91.

25 Spencer R, McMeel JW, Franks EP. Visual outcome in moderate and severe proliferative diabetic retinopathy. Arch Ophthalmol 1981; 99: 1551-4. 